\title{
Depth of invasion determined by MRI in cT1NO tongue: is it an indicator for elective neck dissection?
}

\section{Chunmiao Xu}

Henan Cancer Hospital

Junhui Yuan

Henan Cancer Hospital

Liuqing Kang

Henan Cancer Hospital

Xiaoxian Zhang

Henan Cancer Hospital

Lifeng Wang

Henan Cancer Hospital

Xuejun Chen

Henan Cancer Hospital

Qi Yao

Henan Cancer Hospital

Hailiang Li ( $\square$ lihailiang455@sina.com )

Henan University of Technology https://orcid.org/0000-0001-5792-8342

\section{Research article}

Keywords: tongue squamous cell carcinoma; occult neck lymph node metastasis; depth of invasion; MRI; prognosis

Posted Date: August 28th, 2019

DOI: https://doi.org/10.21203/rs.2.10891/v1

License: (c) (i) This work is licensed under a Creative Commons Attribution 4.0 International License. Read Full License 


\section{Abstract}

Abstracts Background Depth of invasion (DOI) could be calculated by MRI preoperatively, whether MRIdetermined DOI could predict the prognosis and whether it could be used as an indicator for neck dissection for cT1N0 tongue squamous cell carcinoma (SCC) remain unknown, the main goal of the current study aimed to answer the questions. Methods Patients with surgically treated cT1NO tongue SCC were retrospectively enrolled, MRI-determined DOI was measured based on T1-weigthed layers by a 1.5T scan. A multivariate logistic regression analysis model was used to determine the independent predictors for occult neck lymph node metastasis. The main study endpoints were locoregional control survival (LRC) and disease specific survival (DSS), the Cox model was used to determine the independent prognostic factors for the LRC and DSS. Results Occult neck lymph node metastasis was noted in 26 (17.2\%) patients, ROC curve indicated the optimal cutoff value of MRI-determined DOI was $7.5 \mathrm{~mm}$ for predicting neck lymph node metastasis with sensitivity of $86.9 \%$. The factors of lymphovascular invasion, MRI-determined DOI, pathologic DOI, and pathologic tumor grade were significantly associated with the presence of neck lymph node metastasis in univariate analysis, further logistic regression analysis confirmed the independence of lymphovascular invasion, MRI-determined DOI, and pathologic DOI in predicting the neck lymph node metastasis. The 5 -year LRC and DSS rates were $84 \%$ and $90 \%$, respectively. Cox model analysis suggested the MRI-determined DOI was an independent prognostic factor for both the LRC and DSS. Conclusions Elective neck dissection is suggested if MRI-determined DOI is greater than $7.5 \mathrm{~mm}$ in cT1 NO tongue SCC, and MRI-determined DOI $\geq 7.5 \mathrm{~mm}$ indicates more risk for disease recurrence and cancer caused death.

\section{Background}

Neck lymph node metastasis is one of the most important prognostic factors in tongue squamous cell carcinoma (SCC) [1], but unfortunately these positive lymph nodes are usually occult or subclinical at the initial treatment in early stage tongue SCC. Owing to a wide range of occult metastasis rate [2, 3], either elective neck dissection (END) or the watchful waiting policy has been the favored treatment for cT1N0 tongue SCC [4, 5]. Investigators favoring for END comment that END allows more accurate disease stage and decision of the need for adjuvant therapies, and resection of metastatic lymph nodes could potentially reduce the recurrence risk [6,7], however, the main concern according to the traditional watchful waiting policy is the associated surgical complication including shoulder dysfunction and overtreatment for those patients having no pathologic metastases [8]. Considering there is no accurate diagnostic procedure for staging the neck preoperatively, the elective management of the neck in cT1NO tongue SCC has been the subject of much debate during the past 3 decades and continues to be controversial.

Depth of invasion (DOI) is now added in the newest edition of AJCC tumor-node-metastasis staging system [9], and abundant literature has showed the significant relationship between DOI and neck lymph node metastasis [10-12], however, data regarding pathologic DOI usually can not be obtained by frozen section or incisional biopsy, it might has limited role on benefiting decision making of neck treatment 
preoperatively. MRI has been widely used to evaluate the soft tissue disease, and current evidence has reported the reliability of MRI in measuring the DOI $[13,14]$ as well as the prognostic value of MRIdetermined tumor thickness in tongue SCC $[15,16]$, the MRI-determined DOI is significantly different from the MRI-determined tumor thickness, but whether MRI-determined DOI has the same effect with the MRIdetermined tumor thickness and whether it could be used as an indicator for END for CT1NO tongue SCC remain unknown, therefore, the main goal of current study was to clarify these questions.

\section{Patients and methods}

The Zhengzhou University Institutional Research Committee approved our study, and all patients signed informed consent agreements for medical research before initial treatment. All methods were performed in accordance with the relevant guidelines and regulations.

From January 2010 to December 2016, medical records of adult ( $\geq 18$ years) patients with surgically treated tongue SCC were reviewed. Enrolled patients must meet the following criteria: the disease must be primary; the disease was re-staged as CT1NOMO according to the $7^{\text {th }}$ AJCC classification followed by examinations of ultrasound, $C T$, and MRI; Data regarding MRI could be obtained; data regarding the follow-up could be obtained. Information including age, sex, tumor growth pattern, adverse pathologic characteristics, and follow-up of enrolled patients was extracted and analyzed.

MRI-determined DOI was measured based on T1-weigthed layers by a $1.5 \mathrm{~T}$ scan [13, 14], it was defined as the vertical distance between the deepest point of tumor infiltration and the simulated normal mucosal junction (Figure 1). For exogenous tumors, the part above the mucosal surface was neglected, and for ulcerative tumors, the invaginated part was added [17]. The MRI-determined DOI was measured by at least two radiologists.

All pathologic sections were re-reviewed by at least two pathologists, and perineural invasion was considered to be present if tumor cells were identified within the perineural space and/or nerve bundle; lymphovascular infiltration was positive if tumor cells were noted within the lymphovascular channels [18]. The pathologic DOI was measured from the level of the adjacent normal mucosa to the deepest point of tumor infiltration, regardless of the presence or absence of ulceration [9].

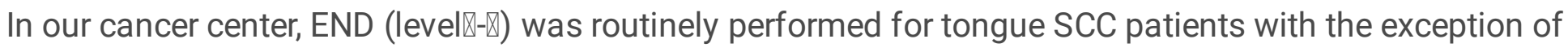
very early-stage disease. Primary tumor excision was usually performed without lip-splitting, the mouth floor tissue was usually preserved unless there was lingual lymph node metastasis reported by frozen section. After therapy, the patients were examined every 3 months during the first year, every 6 months during the second year, and once per year after the second year [1]. Once there was doubt of disease recurrence, aspiration biopsy or incisional biopsy combined other examinations were performed.

The MRI-determined and pathologic DOI was compared using the pair student $t$ test. The ROC curve was used to determine the optimal cutoff of the MRI-determined DOI for predicting the neck lymph node metastasis. The Chi-square test was used to evaluate the association between clinical pathologic 
variables and neck lymph node metastasis, the factors which were significant in the Chi-square test were then analyzed in a multivariate logistic regression analysis model to determine the independent predictors. The primary outcome was locoregional control survival (LRC) and disease specific survival (DSS), the survival time of LRC was calculated from the date of surgery to the date of local or regional recurrence or the last follow-up, and the survival time of DSS was calculated from the date of surgery to the date of cancer-caused death or the last follow-up. The Kaplan-Meier method (log-rank test) was used to calculate the LRC and DSS rates. The factors which were significant in univariate analysis were then analyzed in the Cox model to determine the independent prognostic factors. A value of $p<0.05$ was considered significant, and all statistical analyses were performed with SPSS 20.0.

\section{Methods}

The Zhengzhou University Institutional Research Committee approved our study, and all patients signed informed consent agreements for medical research before initial treatment. All methods were performed in accordance with the relevant guidelines and regulations.

From January 2010 to December 2016, medical records of adult ( $\geq 18$ years) patients with surgically treated tongue SCC were reviewed. Enrolled patients must meet the following criteria: the disease must be primary; the disease was re-staged as CT1NOMO according to the $7^{\text {th }}$ AJCC classification followed by examinations of ultrasound, $\mathrm{CT}$, and MRI; Data regarding MRI could be obtained; data regarding the follow-up could be obtained. Information including age, sex, tumor growth pattern, adverse pathologic characteristics, and follow-up of enrolled patients was extracted and analyzed.

MRI-determined DOI was measured based on T1-weigthed layers by a $1.5 \mathrm{~T}$ scan [13, 14], it was defined as the vertical distance between the deepest point of tumor infiltration and the simulated normal mucosal junction (Figure 1). For exogenous tumors, the part above the mucosal surface was neglected, and for ulcerative tumors, the invaginated part was added [17]. The MRI-determined DOI was measured by at least two radiologists.

All pathologic sections were re-reviewed by at least two pathologists, and perineural invasion was considered to be present if tumor cells were identified within the perineural space and/or nerve bundle; lymphovascular infiltration was positive if tumor cells were noted within the lymphovascular channels [18]. The pathologic DOI was measured from the level of the adjacent normal mucosa to the deepest point of tumor infiltration, regardless of the presence or absence of ulceration [9].

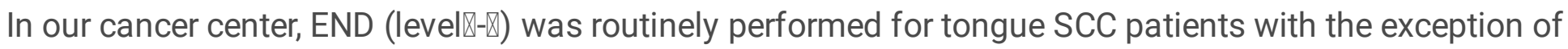
very early-stage disease. Primary tumor excision was usually performed without lip-splitting, the mouth floor tissue was usually preserved unless there was lingual lymph node metastasis reported by frozen section. After therapy, the patients were examined every 3 months during the first year, every 6 months during the second year, and once per year after the second year [1]. Once there was doubt of disease recurrence, aspiration biopsy or incisional biopsy combined other examinations were performed. 
The MRI-determined and pathologic DOI was compared using the pair student $\mathrm{t}$ test. The ROC curve was used to determine the optimal cutoff of the MRI-determined DOI for predicting the neck lymph node metastasis. The Chi-square test was used to evaluate the association between clinical pathologic variables and neck lymph node metastasis, the factors which were significant in the Chi-square test were then analyzed in a multivariate logistic regression analysis model to determine the independent predictors. The primary outcome was locoregional control survival (LRC) and disease specific survival (DSS), the survival time of LRC was calculated from the date of surgery to the date of local or regional recurrence or the last follow-up, and the survival time of DSS was calculated from the date of surgery to the date of cancer-caused death or the last follow-up. The Kaplan-Meier method (log-rank test) was used to calculate the LRC and DSS rates. The factors which were significant in univariate analysis were then analyzed in the Cox model to determine the independent prognostic factors. A value of $p<0.05$ was considered significant, and all statistical analyses were performed with SPSS 20.0.

\section{Results}

There were 151 patients ( 111 male and 40 female) enrolled in total, the mean age was 57.1 (range: 3078) years. There were 102 (67.5\%) smokers and 61 (40.4\%) drinkers, respectively. Flap reconstruction was performed in $32(21.2 \%)$ patients including 25 submental island flaps and 7 radial forearm flaps.

Perineural invasion and lymphovascular invasion were presented in $23(15.2 \%)$ and 19 (12.6\%) patients, respectively. Pathologic tumor grades were distributed as low in 75 patients, intermediate in 51 patients, and high in 25 patients. Negative margin was achieved in all patients. Tumor growth patterns of ulcer type, invasive type, and exogenous type were noted in 72 (47.7\%), 20 (13.2\%), and 59 (39.1\%) patients, respectively.

Positive neck lymph nodes were noted in $26(17.2 \%)$ patients, there was one positive lymph node in 15 patient, two positive lymph nodes in 7 patient, and three positive lymph nodes in 4 patients. There was no extracapsular spread in all the positive lymph nodes.

The mean MRI-determined and pathologic DOI was 6.9 (range: 2-13) $\mathrm{mm}$ and 4.2 (range: $1.0-10.0$ ) mm, respectively, the difference was significant $(p<0.001)$.

ROC analysis described the optimal cutoff value of MRI-determined DOI was $7.5 \mathrm{~mm}$ for predicting neck lymph node metastasis with the area under the curve being 0.848 ; specificity: $82.0 \%$; sensitivity: $86.9 \%$ (Figure 2).

As described in Table 1, the factors of lymphovascular invasion $(p=0.015)$, MRI-determined DOI $(p=0.007)$, pathologic $D O I(p=0.008)$, and pathologic tumor grade $(p=0.034)$ were significantly associated with the presence of neck lymph node metastasis, further logistic regression analysis confirmed the independence of lymphovascular invasion ( $p=0.022,2.475$ [1.233-4.997]), MRI-determined DOI $(p=0.009$, 2.978 [1.574-7.332]), and pathologic DOI $(p<0.001,3.112$ [1.812-9.668]) in predicting the neck lymph node metastasis. 
During our follow up with mean time of 70.4 (range: 8-103) months, 30 patients underwent adjuvant radiotherapy, 6 patients also underwent adjuvant chemotherapy. Locoregional recurrence occurred in 22 patients, disease-caused death occurred in 13 patients.

The 5-year LRC rate was $84 \%$. With regard to prognostic factors for the LRC, as described in Table 2, the factors of perineural invasion $(p=0.016)$, lymphovascular invasion $(p=0.009)$, MRI-determined DOI $(p<0.001)$, pathologic $D O I(p<0.001)$, and neck lymph node metastasis $(p=0.004)$ were significantly related to the LRC. Further cox model indicated the independence of lymphovascular invasion $(p=0.016$, 2.007 [1.274-5.732]), MRI-determined DOI ( $<<0.001,2.842$ [1.449-7.264]), neck lymph node metastasis $(p=0.035,1.745[1.152-4.221])$ and pathologic DOI $(p<0.001,3.246$ [1.679-8.336]) in predicting the LRC. In patients with MRI-determined $\mathrm{DOI} \geq 7.5 \mathrm{~mm}$, the 5 -year LRC rate was $68 \%$, in patients with MRI-determined Dol $7.5 \mathrm{~mm}$, the 5-year LRC was 90\%, the difference was significant (Figure 3, p<0.001).

The 5-year DSS rate was $90 \%$. With regard to prognostic factors for the DSS, as described in Table 3, the factors of MRI-determined DOI ( $p<0.001)$, pathologic DOI $(p<0.001)$, and neck lymph node metastasis $(p=0.008)$ were significantly related to the LRC. Further cox model indicated the independence of MRIdetermined DOI ( $p<0.001,2.441$ [1.635-5.994]), pathologic DOI $(p<0.001,3.002$ [1.753-6.885]), and neck lymph node metastasis $(p=0.005,2.665$ [1.442-5.322]) in predicting the DSS. In patients with MRIdetermined DOI $\geq 7.5 \mathrm{~mm}$, the 5 -year DSS rate was $73 \%$, in patients with MRI-determined DOI $8.5 \mathrm{~mm}$, the 5 -year LRC was $96 \%$, the difference was significant (Figure 4, $p<0.001$ ).

\section{Discussion}

The most valuable finding in current study was that MRI-determined DOI was significantly associated with the presence of neck lymph node metastasis, it would add nearly 3-fold risk of neck lymph node metastasis if MRI-determined DOI was greater than $7.5 \mathrm{~mm}$, and MRI-determined DOI was an independent prognostic factor for the LRC and DSS. The finding might provide preoperative benefit in neck management in CT1N0 tongue SCC.

The feasibility of measurement of DOI by MRI has been widely analyzed $[13,14,16,17]$. Murakami et al. [13] compared the inter-rater reliability of different methods of DOI measurement by MRI, the authors found the method of the axial invasive portion had excellent inter-rater reliability. The data in current study was also obtained by the axial invasive portion. Lam et al.[19] described that the tumor thickness measured on T1-sequence MRI was $0.8 \mathrm{~mm}$ greater than that measured in pathological sections, but 2 $\mathrm{mm}$ greater on $\mathrm{T} 2$ sequences than that measured in pathological sections on average. Similar finding was also noted by Preda et al.[20]. T1-weighted images were more accurate for measuring the DOI than T2 sequences. DOI in T2 weighted images could be overestimated owing to the inflammation and surround tissue edema. Therefore, in current study, the MRI-determined DOI was obtained based on T1 sequences to increase our reliability. In the other hand, Park et al.[14] reported compared to the data measured in postoperative pathological sections, the DOI on T1 MRI was $1.5 \mathrm{~mm}$ greater, but the mean difference between MRI-determined DOI and pathologic DOI was $2.7 \mathrm{~mm}$ in current study, a little greater 
than previous finding $[14,19,20]$, possible explanation was that only staged T1 tumors were included for analysis, relatively higher extent of tissue shrink existed in smaller tumors.

The presence of neck lymph node metastasis was an important prognostic factor for head and neck SCC $[1,3]$. END was usually an important part in primary operation, but owing to the wide range of occult metastasis rate in CT1N0 tongue SCC [7], the neck management of CT1N0 tongue SCC has been debated over the years remaining its controversy. The ideal treatment for patients with cT1N0 tongue SCC must be balanced between and the possible surgical morbidity and optimal oncological outcomes. The common principle was that NO necks should be treated electively when the occult metastatic rate was more than $20 \%[11,21]$. In current study, the overall occult metastasis rate was $17.2 \%$, but all patients underwent END. There were at least three aspects for explaining this phenomenon: firstly, the high requirement of routine follow-up of wait-and-see policy was usually out of our patients' ability, as described by our previous studies [22, 23], patients in our cancer hospital usually came from low income family and remote districts; secondly, there was abundant evidence indicating that there was often a low salvage rate on disease recurrence in patients who do not have prophylactic therapy of the clinically NO neck [2-5], thirdly, also the most important one, there were no reliable predictors for occult neck lymph node metastasis from previous studies.

A number of researchers had aimed to explore the potential predictors for the occult neck lymph node metastasis. Tumor budding was defined as the presence of small clusters of cancer cells or isolated single cancer cell, it suggested a more aggressive biologic behavior and carried more possibility of migrating to the adjacent stroma. Xie et al.[24] described the tumor budding intensity was significantly associated with occult lymph node metastasis. Systemic inflammatory response could promote tumor cell proliferation, microvascular regeneration, and tumor metastasis, further, the peripheral neutrophil-tolymphocyte ratio (NLR) was an accurate and reliable inflammatory marker. High NLR is thought to be significantly associated with worse survival in solid cancers [25]. Abbate et al.[26] firstly presented there was higher risk for occult neck lymph node metastasis when pre-treatment NLR was greater than 2.93. Loganathan et al.[27] recently reported END should be considered when the tumor thickness exceeds 5 $\mathrm{mm}$ based on the significant relationship between tumor thickness and occult neck lymph node metastasis. Other analyzed variables included perineural invasion, lymphovascular invasion, and pathologic DOI $[28,29]$. However, data regarding the pathologic factors usually could not be obtained preoperatively, and pretreatment NLR were nonspecific parameters because they could be influenced by concomitant conditions, such as infections or inflammation. Therefore, more accurate indicators were needed.

As discussed as above, MRI-determined DOI could be reliably calculated preoperatively, and our result presented high predictive value of MRI-determined $\mathrm{DOI} \geq 7.5 \mathrm{~mm}$ in identifying occult metastasis with sensitivity of $86.9 \%$. In another study by Jung et al.[30], the authors recommended a cut-off value of 10.5 $\mathrm{mm}$ in contrast-enhanced T1-weighted images and showed a significant correlation with nodal metastasis, but the authors failed to give the information about the sensitivity, and the variation from ours could be explained by the different calculation method of the cut-off value. The potential 
mechanism for our interesting finding was presented as follows: MRI-determined DOI could indirectly reflect the pathologic DOI, mean difference between MRI-determined DOI and pathologic DOI was $2.7 \mathrm{~mm}$ in current study, therefore, a MRI-determined DOI cut-off value of $7.5 \mathrm{~mm}$ would be indicating a pathologic DOI cut-off value of $5.0 \mathrm{~mm}$, extensive literature had reported the neck lymph node metastasis risk increased apparently if there was pathologic DOI >5.0mm [10, 11, 28, 29].

Prognostic factors for tongue SCC had been extensively analyzed, widely accepted risk factors included disease stage, tumor differentiation, perineural invasion, lymphovascular invasion, neck lymph node status, pathologic DOI, and so on $[1,12,16,23,27,31]$. Similar finding was also noted in current study. But the significance of MRI-determined DOI in the survival of tongue SCC remained unknown, this was the first study to describe a significant association between MRI-determined DOI and the prognosis, MRIdetermined $\mathrm{DO} \geq 7.5 \mathrm{~mm}$ mean higher risk of disease recurrence and cancer-caused death. The potential mechanism might be explained by that greater MRI-determined DOI indicated greater pathologic DOI, the negative affect of pathologic DOI on the prognosis had been widely suggested. Tam et al.[12] recently reported the DOI was an independent predictor for both overall survival and DSS. Similar finding was also presented by lida et al.[32] and Jung et al.[30].

Almost all the literature regarding MRI-determined DOI is just focused on evaluating the association between MRI-determined DOI and pathologic DOI, we hope the current research could provide assistance in neck management in patients with CT1NO tongue SCC and looking for better ways to analyze and control its progression.

Limitation of this study must be acknowledged: firstly, there was inherent bias in a retrospective study. Second, the sample size was relatively small, possibly reducing the statistical power, therefore, larger sample-size studies were needed to clarify the question.

In summary, there is significant relationship between MRI-determined DOI and occult neck lymph node metastasis in cT1NO tongue SCC, elective neck dissection is suggested if MRI-determined DOI is greater than $7.5 \mathrm{~mm}$, and $\mathrm{MRI}$-determined $\mathrm{DOI} \geq 7.5 \mathrm{~mm}$ indicates more risk for disease recurrence and cancer caused death.

\section{Abbreviations}

LRC: locoregional control survival;

SCC: squamous cell carcinoma;

DOI: depth of invasion;

DSS: disease specific survival;

\section{Declarations}




\section{Ethics approval and consent to participate}

The Zhengzhou University institutional research committee approved our study and all participants signed an informed consent agreement for medical research before initial treatment. And all the related procedures were consistent with Ethics Committee regulations.

Consent to publishAll the material came from our cancer center, and the publish consent have been obtained from all the patients.Availability of data and materials

All data generated or analyzed during this study are included in this published article. And the primary data could be achieved from the corresponding author.

Competing interestsThe authors declare that they have no competing interests. FundingThis study was not funded by any outside source.Authors' Contributions

Study design and manuscript writing: CM-X, JH-Y, LQ-K, XX-Z, LF-W, XJ-C, HL-L. Studies selecting and data analysis: CM-X, JH-Y, LQ-K. Study quality evaluating: CM-X, LF-W, XJ-C, HL-L, QY. Manuscript revising: CM$X, J H-Y, L Q-K, X X-Z, L F-W, X J-C, H L-L$. All authors have read and approved the final manuscript.

AcknowledgementsNone declared

\section{References}

1. Fang Q, Li P, Qi J, Luo R, Chen D, Zhang X. Value of lingual lymph node metastasis in patients with squamous cell carcinoma of the tongue. Laryngoscope. 2019. doi: 10.1002/lary.27927.

2. Yuen AP, Wei WI, Wong YM, Tang KC. Elective neck dissection versus observation in the treatment of early oral tongue carcinoma. Head Neck. 1997; 19:583-8.

3. Mücke T, Mitchell DA, Wagenpfeil S, Ritschl LM, Wolff K-D, Kanatas A. Incidence and outcome for patients with occult lymph node involvement in T1 and T2 oral squamous cell carcinoma: a prospective study. BMC Cancer. 2014;14:346.

4. Feng Z, Li JN, Li CZ, Guo CB. Elective neck dissection versus observation in the management of early tongue carcinoma with clinically node-negative neck: a retrospective study of 229 cases. J Craniomaxillofac Surg 2014; 42:806-10.

5. Cao Y, Wang T, Yu C, Guo X, Li C, Li L. Elective neck dissection versus wait-and-watch policy for oral cavity squamous cell carcinoma in early stage: A Systematic Review and Meta-Analysis based on survival data. J. Oral Maxillofac. Surg. 2019 doi: 10.1016/j.joms.2019.03.015.

6. Lim YC, Lee JS, Koo BS, Kim SH, Kim YH, Choi EC. Treatment of contralateral NO neck in early squamous cell carcinoma of the oral tongue: elective neck dissection versus observation. 2006; 116:461-5.

7. Abu-Ghanem S, Yehuda M, Carmel NN, Leshno M, Abergel A, Gutfeld O, Fliss DM. Elective neck dissection vs observation in early-stage squamous cell carcinoma of the oral tongue with no 
clinically apparent lymph node metastasis in the neck: A Systematic Review and Meta-analysis. JAMA Otolaryngol Head Neck Surg. 2016; 142:857-65.

8. Fakih AR, Rao RS, Borges AM, Patel AR. Elective versus therapeutic neck dissection in early carcinoma of the oral tongue. Am J Surg 1989; 158:309-13.

9. Lydiatt WM, Patel SG, O'Sullivan B, Brandwein MS, Ridge JA, Migliacci JC, Loomis AM, Shah JP. Head and Neck cancers-major changes in the American Joint Committee on cancer eighth edition cancer staging manual. CA Cancer J Clin. 2017; 67:122-37.

10. Mair MD, Shetty R, Nair D, Mathur Y, Nair S, Deshmukh A, Thiagarajan S, Pantvaidya G, Lashkar S, Prabhash K, Chaukar D, Pai P, Cruz AD, Chaturvedi P. Depth of invasion, size and number of metastatic nodes predicts extracapsular spread in early oral cancers with occult metastases. Oral Oncol 2018; 81:95-9.

11. Mitani S, Tomioka T, Hayashi R, Ugumori T, Hato N, Fujii S. Anatomic invasive depth predicts delayed cervical lymph node metastasis of tongue squamous cell carcinoma. Am J Surg Patho 2016; 40:93442.

12. Tam S, Amit M, Zafereo M, Bell D, Weber RS. Depth of invasion as a predictor of nodal disease and survival in patients with oral tongue squamous cell carcinoma. Head Neck 2019; 41:177-84.

13. Murakami R, Shiraishi S, Yoshida R, Sakata J, Yamana K, Hirosue A, Uchiyama Y, Nakayama H, Yamashita Y. Reliability of MRI-derived depth of invasion of oral tongue cancer. Acad Radiol 2018. doi: 10.1016/j.acra.2018.08.021.

14. Park JO, Jung SL, Joo YH, Jung CK, Cho KJ, Kim MS. Diagnostic accuracy of magnetic resonance imaging (MRI) in the assessment of tumor invasion depth in oral/oropharyngeal cancer. Oral Oncol 2011; 47:381-6.

15. Okura M, lida S, Aikawa T, Adachi T, Yoshimura N, Yamada T, Kogo M. Tumor thickness and paralingual distance of coronal MR imaging predicts cervical node metastases in oral tongue carcinoma. AJNR Am J Neuroradiol. 2008; 29:45-50.

16. Hu H, Cheng KL, Xu XQ, Wu FY, Tyan YS, Tsai CH, Shen CY. Predicting the prognosis of oral tongue carcinoma using a simple quantitative measurement based on preoperative MR imaging: tumor thickness versus tumor volume. AJNR Am J Neuroradiol 2015; 36:1338-42.

17. Mao MH, Wang S, Feng ZE, Li JZ, Li H, Qin LZ, Han ZX. Accuracy of magnetic resonance imaging in evaluating the depth of invasion of tongue cancer. A prospective cohort study. Oral Oncol 2019; 91:79-84.

18. Skulsky SL, O'Sullivan B, McArdle O, Leader M, Roche M, Conlon PJ, O'Neill JP. Review of high-risk features of cutaneous squamous cell carcinoma and discrepancies between the American Joint Committee on Cancer and NCCN Clinical Practice Guidelines In Oncology. Head Neck 2017; 39:57894.

19. Lam P, Au-Yeung KM, Cheng PW, Wei WI, Yuen AP, Trendell-Smith N, Li JH, Li R. Correlating MRI and histologic tumor thickness in the assessment of oral tongue cancer. AJR Am J Roentgenol. 2004; 182:803-8. 
20. Preda L, Chiesa F, Calabrese L, Latronico A, Bruschini R, Leon ME, Renne G, Bellomi M. Relationship between histologic thickness of tongue carcinoma and thickness estimated from preoperative MRI. Eur Radiol. 2006; 16:2242-8.

21. Weiss MH, Harrison LB, Isaacs RS. Use of decision analysis in planning a management strategy for the stage NO neck. Arch Otolaryngol Head Neck Surg. 1994; 120:699-702.

22. Liu F, Yuan S, Fang Q, Sun Q. Natural history of untreated squamous cell carcinoma of the head and neck. Clin Otolaryngol. 2019; 44:200-3

23. Fang Q, Du W, Jin X, Luo R. Prognostic value of a family history of tongue squamous cell carcinoma: a matched-pair study. Front Oncol 2019 [accepted].

24. Xie N, Wang C, Liu X, Li R, Hou J, Chen X, Huang H. Tumor budding correlates with occult cervical lymph node metastasis and poor prognosis in clinical early-stage tongue squamous cell carcinoma. J Oral Pathol Med. 2015; 44:266-72.

25. Fang Q, Liu F, Seng D. Oncologic outcome of parotid mucoepidermoid carcinoma in pediatric patients. Cancer Manag Res. 2019; 11:1081-5.

26. Abbate V, Dell'Aversana Orabona G, Salzano G, Bonavolontà P, Maglitto F, Romano A, Tarabbia F, Turri-Zanoni M, Attanasi F, Di Lauro AE, laconetta G6, Califano L. Pre-treatment Neutrophil-toLymphocyte Ratio as a predictor for occult cervical metastasis in early stage (T1-T2 cNO) squamous cell carcinoma of the oral tongue. Surg Oncol. 2018; 27:503-7.

27. Loganathan P, Sayan A, Hsu DWK, Paraneetharan S, llankovan V. Squamous cell carcinoma of the anterior tongue: is tumour thickness an indicator for cervical metastasis? Int J Oral Maxillofac Surg. 2017; 46:407-12.

28. Lim SC, Zhang S, Ishii G, Endoh Y, Kodama K, Miyamoto S, Hayashi R, Ebihara S, Cho JS, Ochiai A. Predictive markers for late cervical metastasis in stage I and II invasive squamous cell carcinoma of the oral tongue. Clin Cancer Res. 2004; 10(1 Pt 1):166-72.

29. Sparano A, Weinstein G, Chalian A, Yodul M, Weber R. Multivariate predictors of occult neck metastasis in early oral tongue cancer. Otolaryngol Head Neck Surg. 2004; 131:472-6.

30. Jung J, Cho NH, Kim J, Choi EC, Lee SY, Byeon HK, Park YM, Yang WS, Kim SH. Significant invasion depth of early oral tongue cancer originated from the lateral border to predict regional metastases and prognosis. Int J Oral Maxillofac Surg. 2009; 38:653-60.

31. Dai L, Fang Q, Li P, Wu J, Zhang X. Secondary squamous cell carcinoma of the oral cavity after nasopharyngeal carcinoma. Cancer Res Treat. 2019 [accepted]

32. lida Y, Kamijo T, Kusafuka K, Omae K, Nishiya Y, Hamaguchi N, Morita K, Onitsuka T. Depth of invasion in superficial oral tongue carcinoma quantified using intraoral ultrasonography. Laryngoscope. 2018; 128:2778-2.

\section{Tables}

Table 1. Univariate and multivariate analysis of predictors for neck lymph node metastasis 


\begin{tabular}{|c|c|c|c|c|c|}
\hline \multirow[t]{2}{*}{ Variables } & \multicolumn{2}{|c|}{ Neck lymph node metastasis } & \multirow{2}{*}{$\begin{array}{c}\text { Univariate } \\
\qquad \mathrm{p}\end{array}$} & \multicolumn{2}{|c|}{ Logistic regression } \\
\hline & Positive & Negative & & $\mathrm{p}$ & $\mathrm{OR}[95 \% \mathrm{CI}]$ \\
\hline \multicolumn{6}{|l|}{ Age } \\
\hline$\geq 57$ & 16 & 74 & & & \\
\hline$\square 57$ & 10 & 51 & 0.825 & & \\
\hline \multicolumn{6}{|l|}{ Sex } \\
\hline Male & 20 & 91 & & & \\
\hline Female & 6 & 34 & 0.665 & & \\
\hline \multicolumn{6}{|l|}{ Smokers } \\
\hline Yes & 18 & 84 & & & \\
\hline No & 8 & 41 & 0.841 & & \\
\hline \multicolumn{6}{|l|}{ Drinkers } \\
\hline Yes & 11 & 50 & & & \\
\hline No & 15 & 75 & 0.827 & & \\
\hline \multicolumn{6}{|l|}{ Perineural invasion } \\
\hline Yes & 6 & 17 & & & \\
\hline No & 20 & 108 & 0.221 & & \\
\hline \multicolumn{6}{|c|}{ Lymphovascular invasion } \\
\hline Yes & 7 & 12 & & & \\
\hline No & 19 & 113 & 0.015 & 0.022 & $2.475[1.233-4.997]$ \\
\hline \multicolumn{6}{|l|}{ Pathologic tumor grade } \\
\hline Low & 8 & 67 & & & \\
\hline Intermediate + high & 18 & 58 & 0.034 & 0.110 & 2.414 [0.894-6.114] \\
\hline \multicolumn{6}{|l|}{ MRI-determined DOI* } \\
\hline$\geq 7.5 \mathrm{~mm}$ & 12 & 26 & & & \\
\hline 口7.5mm & 14 & 99 & 0.007 & 0.009 & 2.978 [1.574-7.332] \\
\hline \multicolumn{6}{|l|}{ Pathologic DOI } \\
\hline$\geq 5.0 \mathrm{~mm}$ & 13 & 30 & & & \\
\hline प5.0mm & 13 & 95 & 0.008 & $<0.001$ & 3.112 [1.812-9.668] \\
\hline \multicolumn{6}{|l|}{ Tumor growth pattern } \\
\hline Ulcer type & 12 & 60 & & & \\
\hline Invasive type & 5 & 15 & & & \\
\hline Exogenous type & 9 & 50 & 0.599 & & \\
\hline
\end{tabular}

*: DOI: depth of invasion.

Table 2. Prognostic factors for the locoregional control survival in patients with T1 tumors. 


\begin{tabular}{lccc}
\hline Variables & Univariate & & Cox model \\
& Log-rank test & $\mathrm{p}$ & RR[95\%CI] \\
Age & 0.634 & & \\
Sex & 0.187 & & \\
Smokers & 0.334 & & \\
Drinkers & 0.227 & & \\
Neck lymph node metastasis & 0.004 & 0.035 & 1.745 [1.152-4.221] \\
Perineural invasion & 0.016 & 0.114 & \\
Lymphovascular invasion & 0.009 & 0.016 & $2.007[1.274-5.732]$ \\
Pathologic tumor grade & 0.095 & & \\
MRI-determined DOI & $<0.001$ & $<0.001$ & 2.842 [1.449-7.264] \\
Pathologic DOI & $<0.001$ & $<0.001$ & 3.246 [1.679-8.336] \\
Tumor growth pattern & 0.397 & & \\
Adjuvant treatment & 0.572 & & \\
\hline
\end{tabular}

Table 3. Prognostic factors for the disease-specific survival in patients with T1 tumors.

\begin{tabular}{lccc}
\hline Variables & Univariate & & Cox model \\
& Log-rank test & $\mathrm{p}$ & RR[95\%CI] \\
Age & 0.241 & & \\
Sex & 0.387 & & \\
Smokers & 0.841 & & \\
Drinkers & 0.458 & & \\
Neck lymph node metastasis & 0.008 & 0.005 & 2.665 [1.442-5.322] \\
Perineural invasion & 0.089 & & \\
Lymphovascular invasion & 0.110 & & \\
Pathologic tumor grade & 0.175 & & \\
MRI-determined DOI & $<0.001$ & $<0.001$ & 2.441 [1.635-5.994] \\
Pathologic DOI & $<0.001$ & $<0.001$ & 3.002 [1.753-6.885] \\
Tumor growth pattern & 0.422 & & \\
Adjuvant treatment & 0.631 & & \\
\hline
\end{tabular}

\section{Figures}




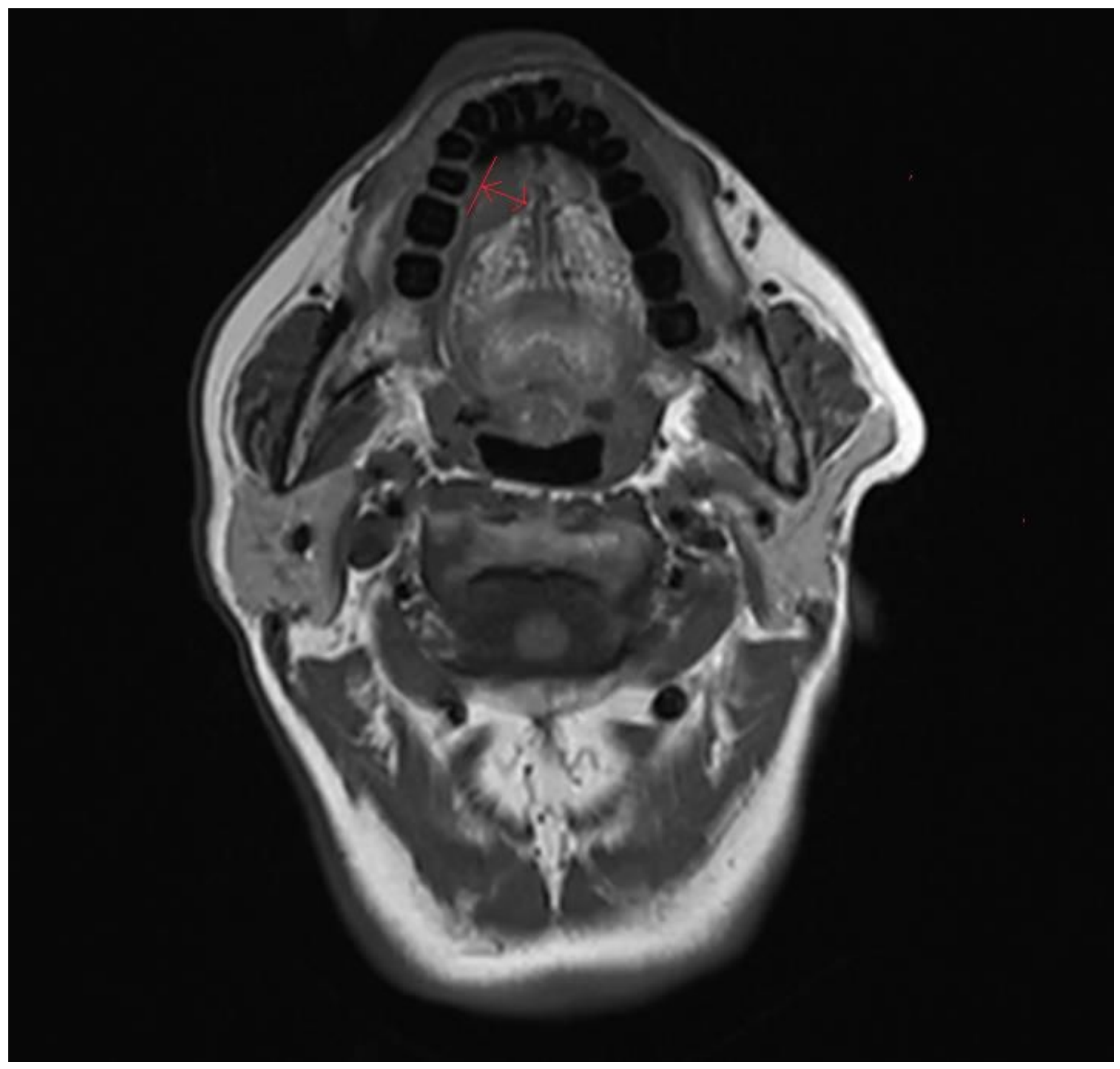

\section{Figure 1}

Measurement of the MRI-determined depth of invasion based on the adjacent normal mucosal junction to the deepest infiltration point. 


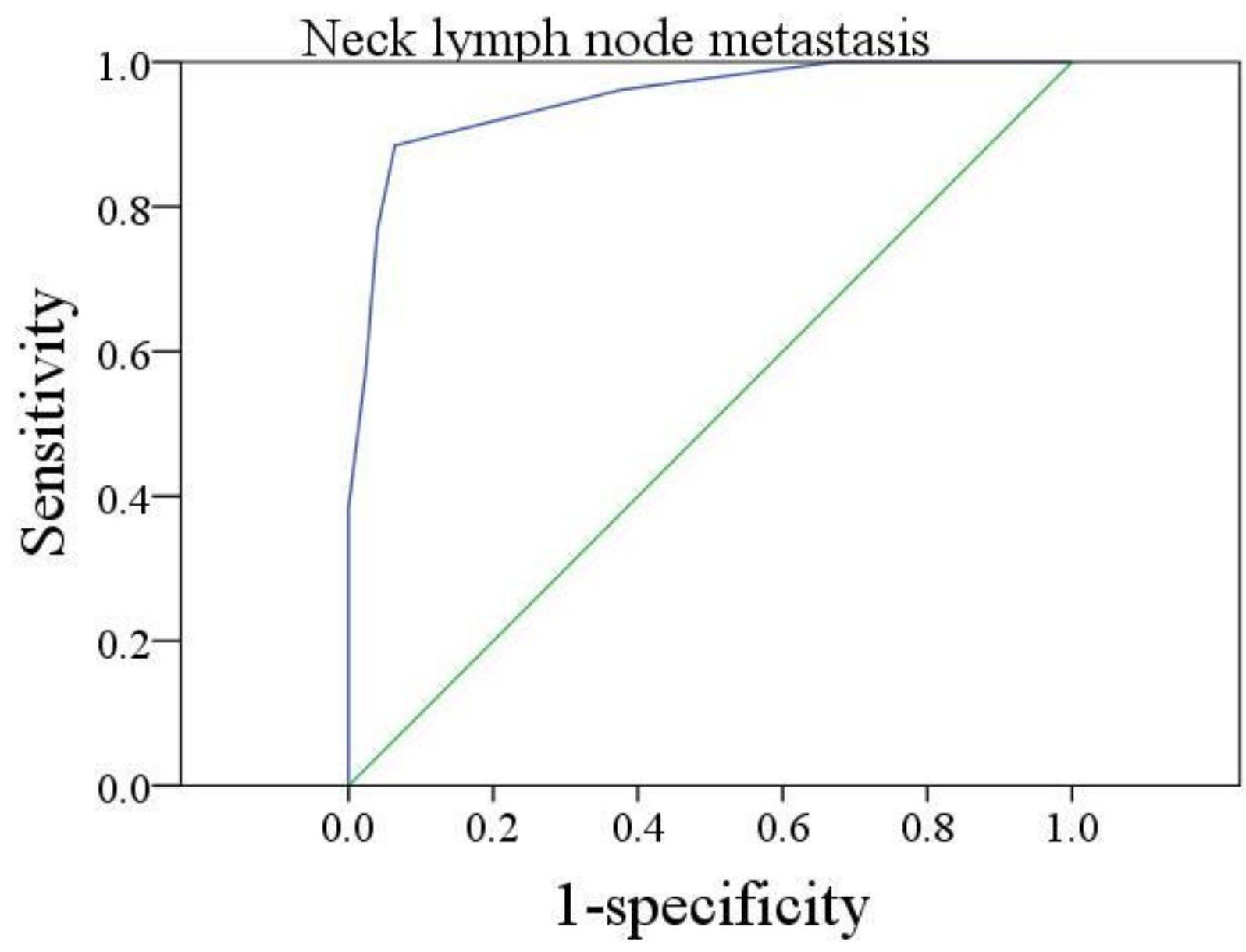

Figure 2

ROC analysis of the optimal cutoff value of MRI-determined DOI for predicting neck lymph node metastasis. 


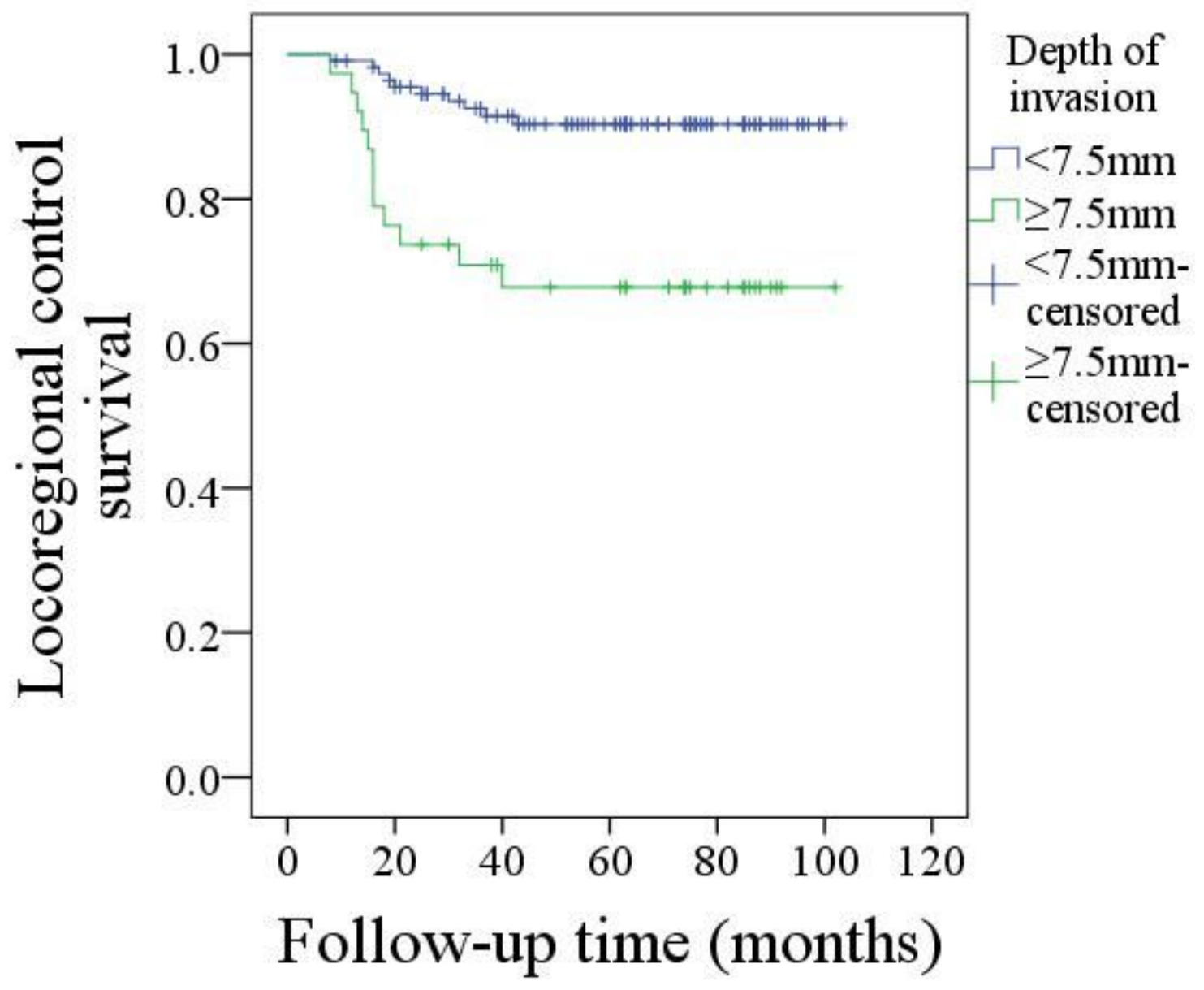

Figure 3

Comparison of locoregional control survival in patients with different MRI-determined depth of invasion $(p<0.001)$. 


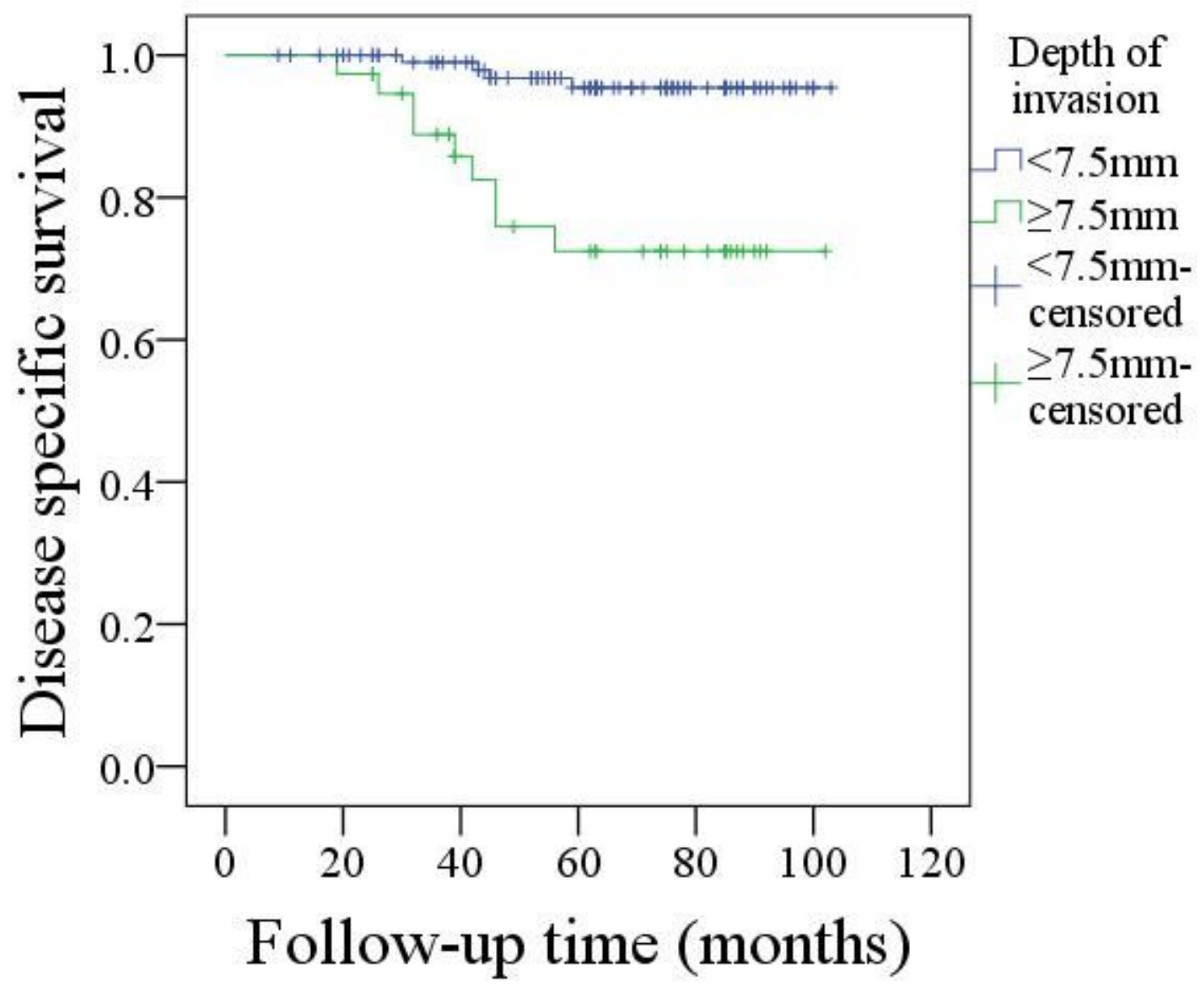

Figure 4

Comparison of disease specific survival in patients with different MRI-determined depth of invasion $(p<0.001)$. 